\title{
Monomolecular Siloxane Film as a Model of Single
}

\section{Site Catalysts}

Michael W. Martynowycz ${ }^{\dagger \S}$, Bo H $u^{\ddagger}$, Ivan Kuzmenko ${ }^{\S}$,Wei Bu ${ }^{\perp}$, Adam Hock $k^{\S \ddagger}$, and David Gidalevitz $^{\dagger *}$

${ }^{\dagger}$ Center for the Molecular Study of Condensed Soft Matter and Department of Physics, Illinois Institute of Technology, Chicago, Illinois, 60616

$\S$ Argonne National Laboratory, Lemont, Illinois, 60439

$\$$ Department of Chemistry, Illinois Institute of Technology, Chicago, Illinois 60616

${ }^{\perp}$ Center for the Advancement of Radiation Sources (CARS), University of Chicago, Chicago, Illinois, 60637

ASSOCIATED CONTENT

Supporting Information. 


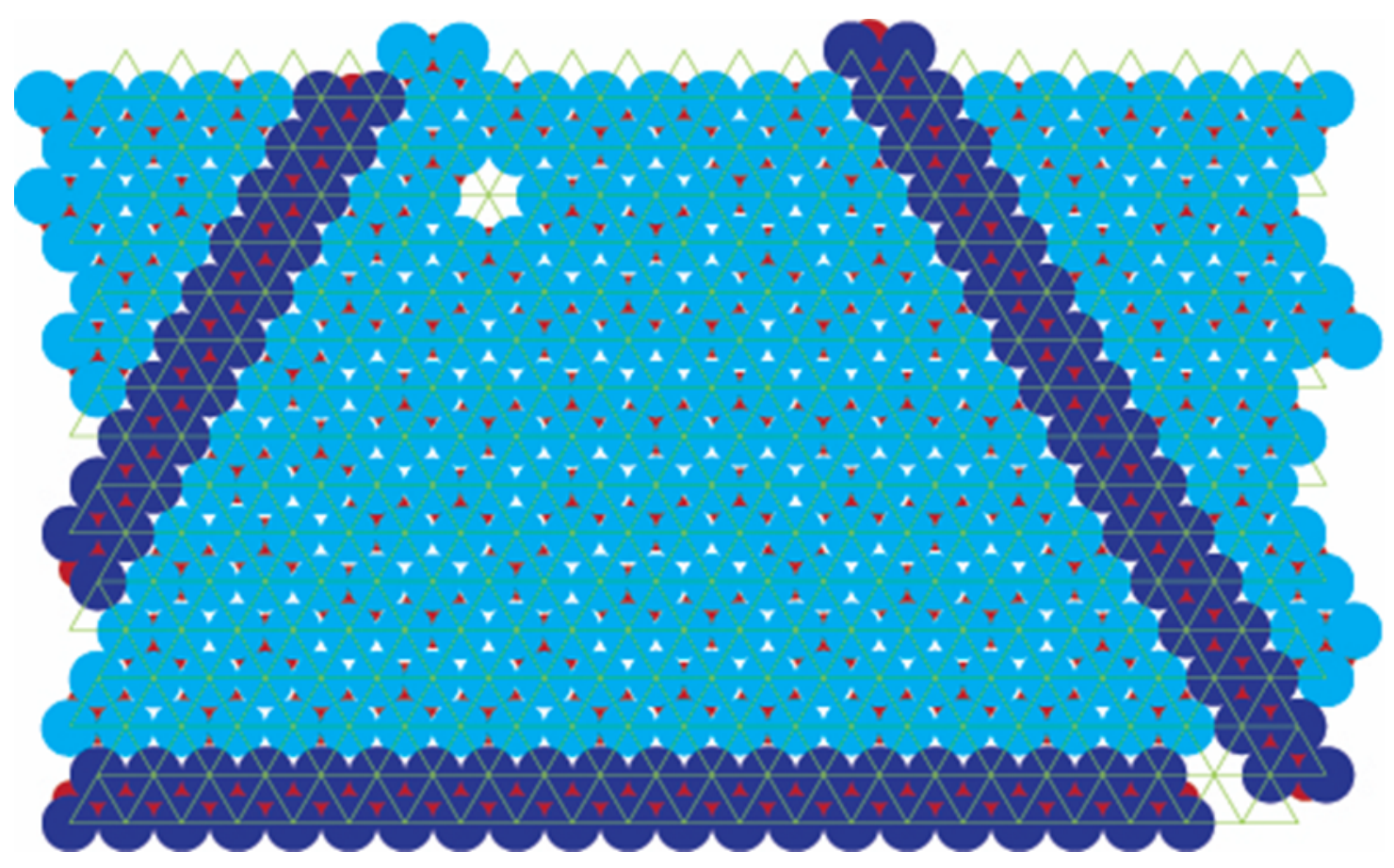

Supplementary Figure-1: A scheme of composite crystal structure of OTOS monolayer that is formed mostly by the cyclic trimers with some amount of linear OTOS polymers or oligomers located at the boundaries (hydrocarbon chains marked in dark blue). The packing constrains would likely lead to point defects (white spot). 


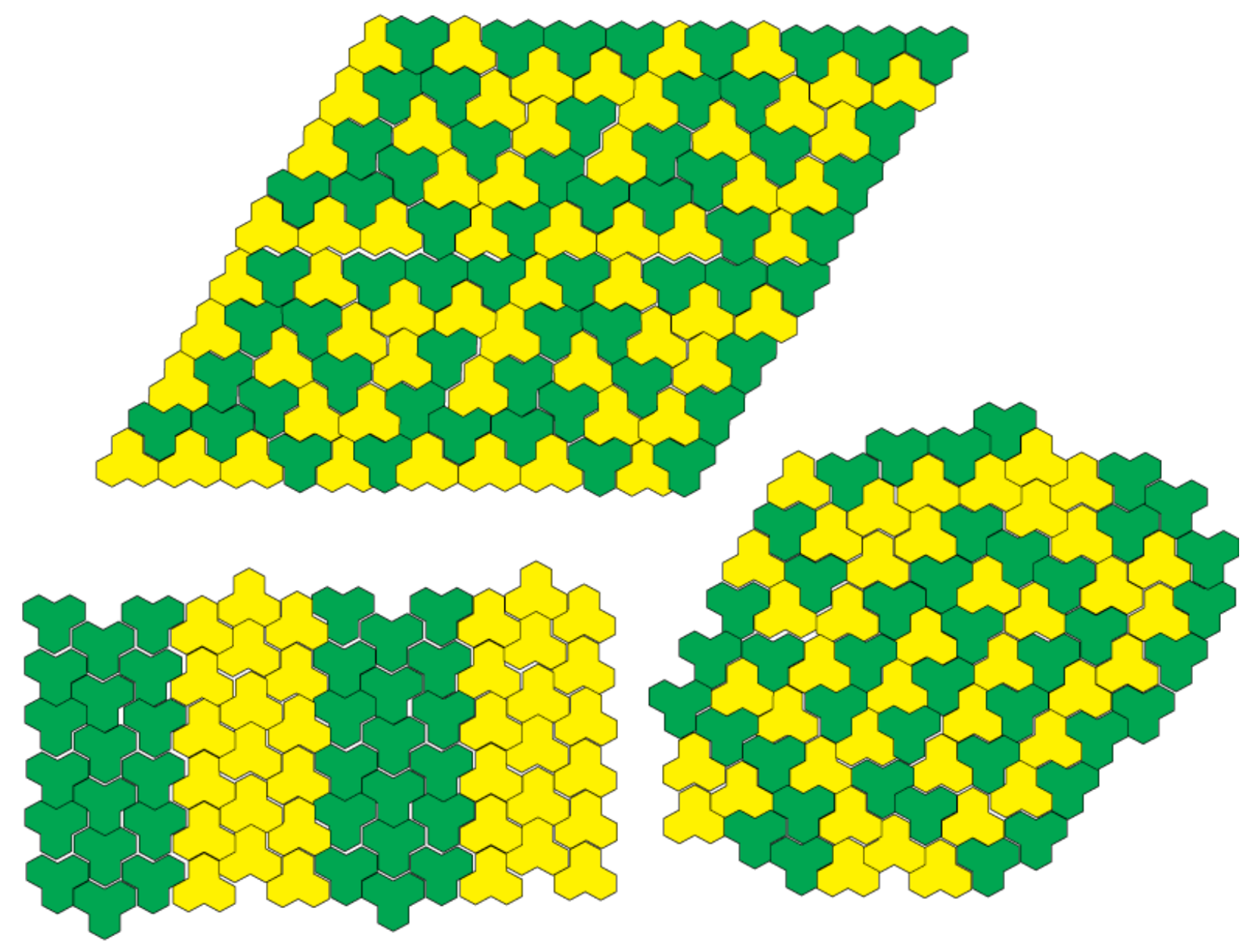

Supplementary Figure-2: Examples of periodic and non-periodic tessellation schemes of the OTOS trimer-packing motifs. 


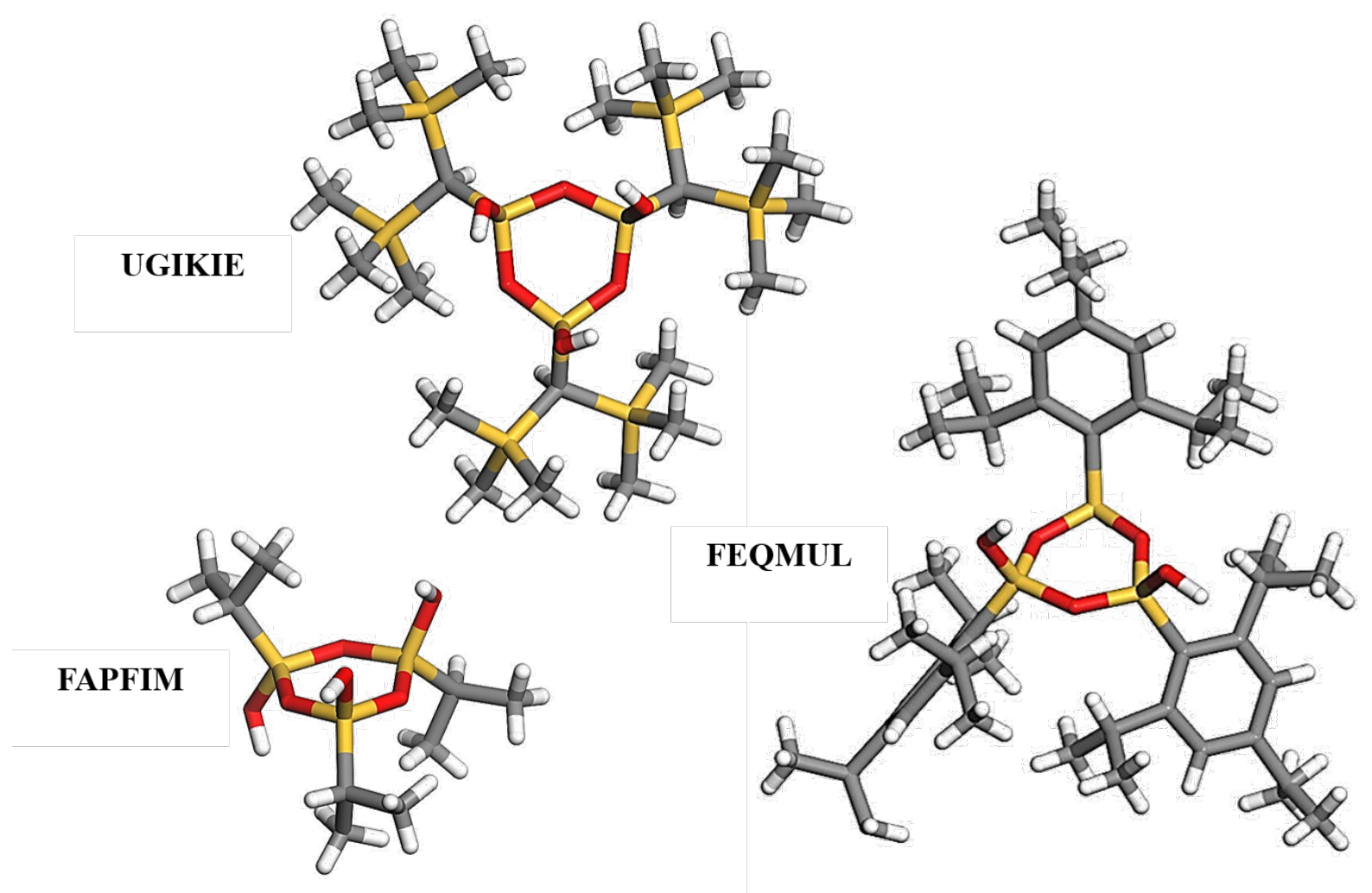

Supplementary Figure-3: Three structural examples of organosilanes, retrieved from the Cambridge Structural Database and labeled with the corresponding refcodes. All structures contain a six-membered Si-O ring; UGIKIE is the closest structural analog of the OTOS trimer model having three hydroxyl groups in cis-orientations.

Supplementary Table-1: Averaged measurements from Cambridge Structural Database for organosilanes. List of all measures found in attached excel document.

\begin{tabular}{ccc}
\hline Measure & Average & Std Deviation \\
\hline Si...Si distance $(\AA)$ & $3.103870599 \AA$ & $0.05570164 \AA$ \\
Si-O distance $(\AA)$ & $1.620459978 \AA$ & $0.022739985 \AA$ \\
Si-O-Si angle $\left({ }^{\circ}\right)$ & $147.4836037^{\circ}$ & $7.81452182^{\circ}$ \\
\hline
\end{tabular}



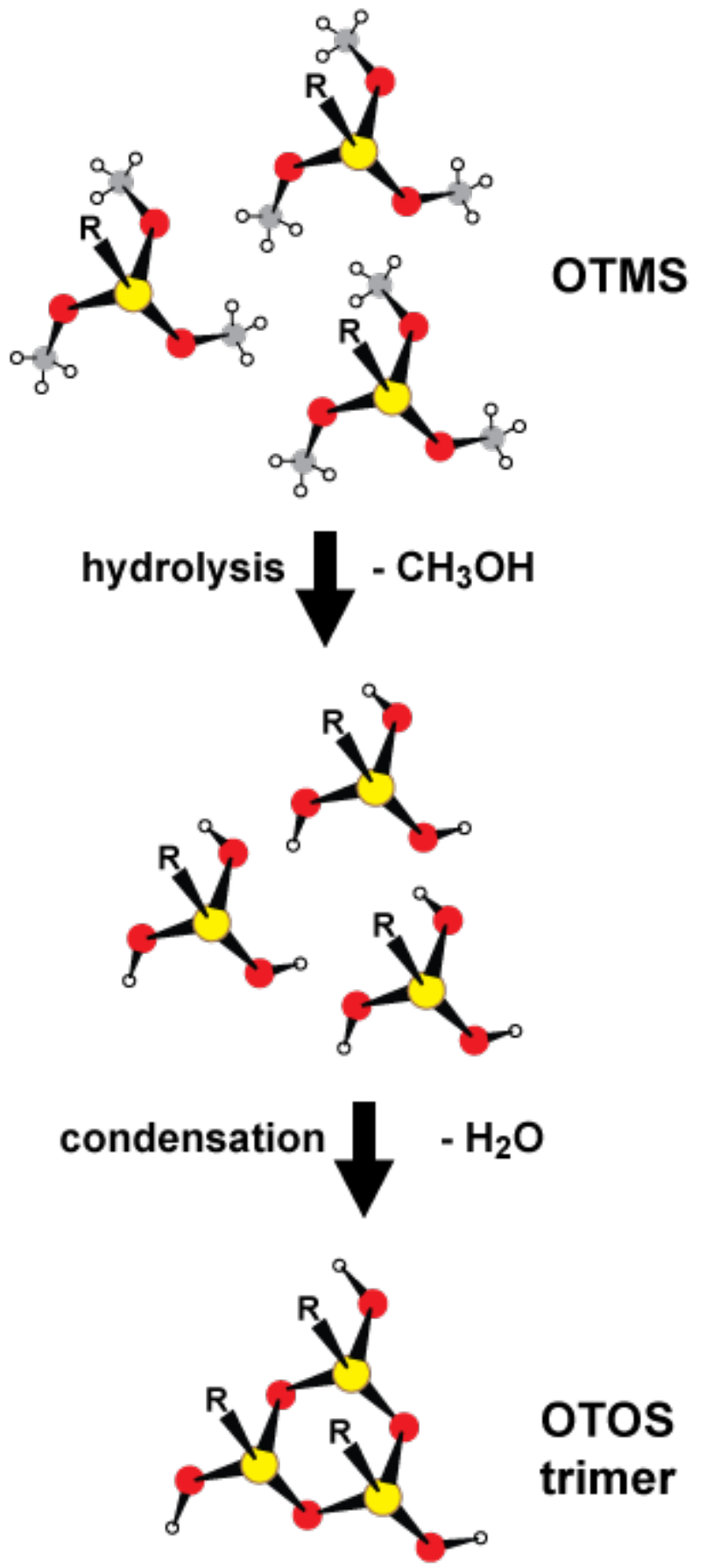

Supplementary Figure 4: Schematic cartoon of the condensation of the OTMS monomers into OTOS trimers at the air-liquid interface. 